\title{
AMP-activated protein kinase controls lipid and lactose synthesis in bovine mammary epithelial cells
}

\author{
J. Huang, M.-A. Guesthier, and S. A. Burgos* (1) \\ Department of Animal Science, McGill University, Sainte-Anne-de-Bellevue, QC, H9X 3V9 Canada
}

\begin{abstract}
The synthesis of milk components in bovine mammary epithelial cells (BMEC) requires an adequate supply of energy. The AMP-activated protein kinase (AMPK) is a cellular energy gauge that controls anabolic and catabolic processes to maintain a balance between energy supply and demand. The objectives of this study were to assess the role of AMPK on de novo lipid and lactose synthesis, as well as its regulation by glucose and acetate availability in BMEC. We isolated primary BMEC from the mammary tissue of 3 lactating Holstein cows and differentiated them with lactogenic hormones for $4 \mathrm{~d}$. We measured protein abundance, site-specific phosphorylation, and proteolytic processing by immunoblotting. We quantified the expression of genes involved in lipid and lactose synthesis using real-time quantitative PCR. We measured de novo lipid and lactose synthesis by incorporation of radioactive substrates. We analyzed data by ANOVA using a randomized complete block design with PROC MIXED in SAS. To assess the effect of AMPK activation on milk component synthesis, we treated BMEC with 100 $\mu M \mathrm{~A}-769662$ (A76; an allosteric activator of AMPK) or vehicle control for $16 \mathrm{~h}$. Consistent with activation of AMPK, A76 increased phosphorylation of its downstream targets ACC Ser79 and TSC2 Ser1387 by $144 \%$ and $26 \%$, respectively. Activation of AMPK decreased lipid synthesis by $19 \%$. This effect was accompanied by increased expression of FABP3. Activation of AMPK reduced the proportion of mature SREBP-1c. In addition, AMPK activation reduced lactose synthesis by $24 \%$ and lowered the expression of SLC2A1, the gene encoding GLUT1. To assess the regulation of AMPK by energy substrate availability, we incubated BMEC in a control medium containing $4 \mathrm{~m} M \mathrm{D}$-glucose and $1 \mathrm{~m} M$ sodium acetate, or medium lacking glucose or acetate, for $4 \mathrm{~h}$. Compared with the control medium, deprivation of glucose or acetate promoted AMPK $\alpha$ phosphorylation at Thr172 by $84 \%$ or $58 \%$, respec-
\end{abstract}

Received January 20, 2019.

Accepted September 19, 2019.

*Corresponding author: sergio.burgos@mcgill.ca tively. Activation of AMPK was significantly increased in BMEC only when the medium was devoid of glucose for at least $4 \mathrm{~h}$. We concluded that activation of AMPK inhibits de novo lipid and lactose synthesis in BMEC. Further studies are needed to assess the physiological relevance of AMPK activation for milk composition in vivo and to identify the mechanisms mediating its effects on milk component synthesis.

Key words: bovine mammary epithelial cell, AMPactivated protein kinase, lactose synthesis, lipid synthesis

\section{INTRODUCTION}

The AMP-activated protein kinase (AMPK) functions as a cellular energy gauge to maintain a balance between energy supply and demand by controlling anabolic and catabolic processes in cells (Hardie, 2011a). The AMPK is a heterotrimeric complex composed of a catalytic $\alpha$ subunit and 2 regulatory subunits, $\beta$ and $\gamma$. Under conditions of energy shortage that result in an increase in AMP/ATP ratio, AMP binds the $\gamma$ subunit of AMPK, thereby activating the kinase activity of the complex (Hardie et al., 2016). Phosphorylation of the AMPK $\alpha$ subunit at Thr172 is critical for its activation (Hawley et al., 1996). The AMPK is activated by physiological conditions that increase intracellular AMP, including nutrient deprivation, hypoxia, and ischemia. We have previously shown that energy depletion by incubation with the glycolytic inhibitor 2-deoxy-Dglucose inhibits protein synthesis through AMPK in the immortalized bovine mammary epithelial cell line MAC-T (Burgos et al., 2013). These results raised the possibility that AMPK may be implicated in regulating the synthesis of other milk components.

The AMPK regulates lipid metabolism by modifying lipogenic gene transcription and post-translational modification of major enzymes involved in lipid synthesis. The effects of AMPK on lipid metabolism are mediated in part by modulation of the master lipogenic transcription factor SREBP-1c. AMPK phosphorylates SREBP-1c at Ser327 to inhibit its proteolytic processing, thereby reducing the amount of mature SREBP-1c in the nucleus and transcriptional activity ( $\mathrm{Li}$ et al., 
2011). In addition to transcriptional mechanisms, induction of AMPK activity downregulates the rate of fatty acid synthesis and speeds up fatty acid oxidation via inhibitory phosphorylation of ACC at Ser79 (Carlson and Kim, 1973; Hardie and Pan, 2002); ACC is a specific enzyme that catalyzes the irreversible carboxylation of acetyl-CoA to produce malonyl-CoA in the fatty acid synthesis pathway (Hardie, 2011b). McFadden and Corl (2009) observed a decrease in de novo fatty acid synthesis, despite a significant increase in the mRNA expression of many lipogenic genes (FASN, FABP3, GPAT, SREBF1) and ACC activity in response to the AMP analog AICAR (5-aminoimidazole4-carboxamide riboside) in MAC-T cells. However, the molecular mechanisms mediating the effect of AMPK on lipid synthesis in bovine mammary epithelial cells (BMEC) remain to be elucidated.

Synthesis of milk components requires an adequate supply of energy, as well as precursors for milk proteins, lipids, and lactose to mammary epithelial cells. In ruminants, the main energy-yielding substrates are acetate, BHB, fatty acids, and glucose. In addition, glucose and acetate are substrates for milk lactose and de novo lipid synthesis, respectively. Therefore, synthesis of milk components and cellular energy metabolism must be tightly coupled. Glucose uptake is considered to be a potential mechanism that limits lactose synthesis in the mammary gland. Zhang et al. (2011) showed that glucose uptake and mRNA abundance of GLUT1 were elevated after incubation of goat mammary epithelial cells with AICAR. In the mammary gland, 55 to $70 \%$ of glucose flux is directed to lactose synthesis, with most of the remaining glucose used for ATP production through glycolysis and the tricarboxylic acid cycle (Guinard-Flament et al., 2006). However, despite the clear importance of AMPK in regulating energy flux and nutrient metabolism in a variety of cell types, the role of AMPK in the regulation of glucose metabolism and milk component synthesis has not been fully characterized. We hypothesized that deprivation of energyyielding substrates activates AMPK to regulate lipid and lactose synthesis by modulating the expression of key genes in these metabolic pathways in BMEC. The objective of the present study was to determine the role of AMPK activation on glucose metabolism and lactose synthesis, as well as its regulation by energy-yielding substrate availability in BMEC.

\section{MATERIALS AND METHODS}

\section{Materials}

Chemicals and cell culture reagents were purchased from Sigma-Aldrich (Oakville, ON, Canada) or Ther-
moFisher Scientific (Waltham, MA), respectively, unless otherwise stated. Collagenase type 3 was from Worthington Biochemical Corp. (Lakewood, NJ). We purchased A76 from Toronto Research Chemicals (Toronto, ON, Canada). Primary antibodies were from Cell Signaling Technology (Beverly, MA). Reagents and materials for immunoblotting and real-time quantitative PCR (qPCR) were from Bio-Rad Laboratories (Hercules, CA). We purchased MCDB170 from US Biological (Salem, MA). Radiochemicals were from PerkinElmer (Waltham, MA). The source and catalog numbers of the reagents are listed in Supplemental Table S1; http: //dx.doi.org/10.3168/jds.2019-16343).

\section{Isolation and Culture of BMEC}

Mammary tissue was collected from 3 multiparous lactating Holstein cows upon slaughter at a local abattoir. Mammary acini were prepared essentially as described by Burgos et al. (2010), with minor modifications. Briefly, after exsanguination, slices of tissue were aseptically excised from the mammary gland and placed in ice-cold Dulbecco's modified Eagle medium/ Nutrient Mixture F-12 [DMEM/F12+; containing 1 $\times$ antibiotics/antimycotics $(100 \mathrm{U} / \mathrm{mL}$ penicillin, 100 $\mu \mathrm{g} / \mathrm{mL}$ streptomycin, $0.25 \mu \mathrm{g} / \mathrm{mL}$ amphotericin $\mathrm{B}$, and $50 \mu \mathrm{g} / \mathrm{mL}$ gentamicin)] for immediate transport to the laboratory. Tissue samples were trimmed of visible connective and adipose tissue and then finely minced into $\sim 1-\mathrm{mm}^{3}$ pieces in $10 \mathrm{~mL}$ of Hanks' balanced salt solution containing $1 \times$ antibiotics/antimycotics (HBSS + ). Minced tissue was subsequently transferred to a $50 \mathrm{~mL}$ centrifuge tube and resuspended with ice-cold HBSS+ to a final volume of $50 \mathrm{~mL}$. After inverting to mix the contents, the tube was placed on ice and the tissue was allowed to settle. The supernatant was then aspirated to remove residual milk and blood. This procedure was repeated until the HBSS + appeared clear. The minced tissue was resuspended in an equal volume of tissue dissociation solution (DMEM/F12+, $300 \mathrm{U} / \mathrm{mL}$ collagenase type $3,100 \mathrm{U} / \mathrm{mL}$ hyaluronidase, $0.4 \mu \mathrm{g} / \mathrm{mL}$ DNase I, and $10 \mu \mathrm{g} / \mathrm{mL}$ bovine insulin) and transferred to a baffled flask with a screw cap and spout for incubation at $37^{\circ} \mathrm{C}$ with constant shaking at $200 \mathrm{rpm}$ for $4 \mathrm{~h}$. After filtering through a sterile sieve with $200-\mu \mathrm{m}$ mesh, the tissue digest was transferred into a $50-\mathrm{mL}$ centrifuge tube and centrifuged at $80 \times g$ for $30 \mathrm{~s}$ at room temperature. The supernatant was discarded, and the pellet, which was highly enriched epithelial organoids, was resuspended in 1 to $5 \mathrm{~mL}$ of DMEM/F12+ in a $50-$ $\mathrm{mL}$ centrifuge tube. Mammary organoids were either cryopreserved or seeded into $100 \mathrm{~mm}$ collagen-coated dishes containing M87A+X medium (a serum-reduced medium that selectively stimulates growth of mammary 
epithelial cells; Garbe et al., 2009) to allow for primary mammary epithelial cell outgrowth. The medium was composed of 1:1 DMEM/F12+:MCDB170, 0.25\% (vol/ vol) fetal bovine serum, $0.1 \%$ (wt/vol) albumax II, 7.5 $\mu \mathrm{g} / \mathrm{mL}$ bovine insulin, $0.3 \mu \mathrm{g} / \mathrm{mL}$ hydrocortisone, 5 $\mathrm{ng} / \mathrm{mL}$ recombinant human epidermal growth factor, $2.5 \mu \mathrm{g} / \mathrm{mL}$ bovine apo-transferrin, $5 \mu M$ isoproterenol, $5 \mathrm{p} M 3,3^{\prime}, 5$-triiodo-L-thyronine, $0.5 \mathrm{p} M \beta$-estradiol, 0.1 $\mathrm{n} M$ oxytocin, and $1 \times$ antibiotics/antimycotics. Mammary organoids were fed every 2 to $3 \mathrm{~d}$ in an incubator at $37^{\circ} \mathrm{C}$ with a $5 \% \mathrm{CO}_{2}$ humidified atmosphere until primary mammary epithelial cells were passaged.

All experiments were performed in second-passage BMEC. Cells were seeded on $35 \mathrm{~mm}$ collagen-coated cell culture dishes at $5 \times 10^{4}$ cells $/ \mathrm{cm}^{2}$ and grown to confluence in $\mathrm{M} 87 \mathrm{~A}+\mathrm{X}$ medium at $37^{\circ} \mathrm{C}$ in a humidified atmosphere with $5 \% \mathrm{CO}_{2}$. The BMEC were induced to differentiate for $4 \mathrm{~d}$ in DMEM modified to contain $4 \mathrm{~m} M$ D-glucose, $1 \mathrm{~m} M$ sodium acetate, and $200 \mu M$ L-glutamine supplemented with lactogenic hormones (5 $\mu \mathrm{g} / \mathrm{mL}$ each of bovine insulin, ovine prolactin, and hydrocortisone), $5 \mu \mathrm{g} / \mathrm{mL}$ apo-transferrin, $0.5 \mathrm{mg} / \mathrm{mL}$ BSA, and $1 \times$ antibiotic-antimycotics. The medium was changed on d 2 of differentiation.

\section{Cell Treatments}

The allosteric AMPK activator A-769662 (A76) was dissolved in dimethyl sulfoxide. For treatment, we added A76 or vehicle control (Ctrl) to lactogenic differentiated BMEC to a final concentration of 100 $\mu M$ for $16 \mathrm{~h}$. To test the effect of glucose and acetate on AMPK activation, cells were incubated in DMEM medium containing $4 \mathrm{~m} M$ glucose and $1 \mathrm{~m} M$ sodium acetate (control), or a control medium lacking glucose or acetate, or both, for $4 \mathrm{~h}$. To define the sensitivity of AMPK to glucose levels, BMEC were treated with DMEM without sodium acetate but with 1,2 , or $4 \mathrm{~m} M$ glucose, or without glucose, for $4 \mathrm{~h}$. For time course experiments, cells were incubated in DMEM without glucose for $0,1,2$, or $4 \mathrm{~h}$.

\section{Lipid Synthesis Assay}

Lipid synthesis in BMEC was determined by quantifying the incorporation of $\left[{ }^{3} \mathrm{H}\right]$-labeled acetate into total lipids. After $12 \mathrm{~h}$ of treatment, cells were incubated with $\left[{ }^{3} \mathrm{H}\right]$-labeled acetate $(0.084 \mathrm{Ci} / \mathrm{mmol})$ at a final concentration of $1 \mu \mathrm{Ci} / \mathrm{mL}$ for $4 \mathrm{~h}$. Cells were then lysed in $0.1 \%$ SDS in PBS. A portion of the cell lysate was retained to measure protein concentration. Total lipids were extracted from cell lysates using hexane: isopropanol $(3: 2 \mathrm{vol} / \mathrm{vol})$. The solvent layer was combined with scintillation cocktail (Universol; MP Bio- medical, Solon, $\mathrm{OH}$ ) for quantification of radioactive label incorporation into lipids using a Tri-Carb liquid scintillation counter (PerkinElmer). Lipid synthesis was expressed as counts per minute of acetate incorporated per microgram of protein.

\section{Lactose Synthesis Assay}

Lactose synthesis in BMEC was determined by quantifying the incorporation of radioactive glucose into lactose. Methods were adapted from Kwon et al. (1981) and Mellenberger et al. (1973). After $12 \mathrm{~h}$ of treatment, cells were incubated with $\left[\mathrm{U}_{-}{ }^{14} \mathrm{C}\right]$-D-glucose $(263 \mathrm{mCi}$ / $\mathrm{mmol}$ ) at a final concentration of $0.1 \mu \mathrm{Ci} / \mathrm{mL}$ for $4 \mathrm{~h}$. A portion of the cell lysate was retained to measure protein concentration. Total lactose was extracted from spent media using $10 \mathrm{M}$ solution of $\mathrm{MgCl}_{2}$ and $10 \mathrm{~N}$ $\mathrm{NaOH}$. The precipitate layer was lactose- $\mathrm{Mg}(\mathrm{OH})_{2}$ slurries for quantification of radioactive label incorporation into lactose using a Tri-Carb liquid scintillation counter (PerkinElmer). Lactose synthesis was expressed as counts per minute of glucose incorporated per microgram of protein.

\section{Immunoblotting}

Cells were rinsed once with ice-cold PBS before harvest. Cellular proteins were extracted in ice-cold lysis buffer containing $1 \%$ (vol/vol) Triton X-100, $50 \mathrm{mM}$ Tris-HCl, pH 7.4, $150 \mathrm{~m} M$ NaCl, $1 \mathrm{~m} M$ EDTA, $1 \mathrm{~m} M$ EGTA, $50 \mathrm{~m} M$-glycerophosphate, $50 \mathrm{~m} M$ NaF, 10 $\mathrm{m} M \mathrm{Na}_{4} \mathrm{P}_{2} \mathrm{O}_{7}, 2 \mathrm{~m} M \mathrm{Na}_{3} \mathrm{VO}_{4}$, and $1 \times$ protease inhibitor cocktail on ice for $15 \mathrm{~min}$. Cell lysates were cleared by centrifugation at $15,000 \times g$ at $4^{\circ} \mathrm{C}$ for $10 \mathrm{~min}$. We used an aliquot of cleared cell lysate to measure protein concentration using a BCA protein assay kit with bovine serum albumin as standard, according to the manufacturer's instructions (http://tools.thermofisher .com/content/sfs/manuals/MAN0011430_Pierce_BCA _Protein_Asy_UG.pdf). We mixed an aliquot of cleared cell lysate with $5 \times$ Laemli sample buffer and then heated it at $95^{\circ} \mathrm{C}$ for $5 \mathrm{~min}$. Equal amounts of protein were resolved by SDS-PAGE and then transferred onto polyvinylidene difluoride membranes. The membranes were blocked in $5 \%$ (wt/vol) nonfat milk in Tris-buffered saline containing $0.1 \%$ (vol/vol) Tween 20 (TBS$\mathbf{T})$ at room temperature for $1 \mathrm{~h}$ and then incubated with primary antibodies raised against phospho-specific proteins diluted in $5 \%$ nonfat milk in TBS-T at $4^{\circ} \mathrm{C}$ with constant rocking overnight. After washing 6 times in TBS-T for 5 min, membranes were incubated with secondary antibodies diluted 1:10,000 in 5\% nonfat milk in TBS-T at room temperature for $1 \mathrm{~h}$ with constant shaking. After washing 6 times in TBS-T, the bound 
horseradish peroxidase-linked secondary antibodies were visualized by chemiluminescence (Clarity ECL, Bio-Rad Laboratories). The band signal intensity was quantified using Image Lab Software (Bio-Rad Laboratories). After detection of the phospho-specific signal, the antibodies were stripped off the membranes by incubation in $62.5 \mathrm{~m} M$ Tris-HCl, $\mathrm{pH} 6.8,2 \%$ (wt/vol) SDS, and $100 \mathrm{mM}$ 2-mercaptoethanol at $50^{\circ} \mathrm{C}$ for $30 \mathrm{~min}$ with constant rocking. The membranes were washed, blocked, and re-probed with primary antibodies that recognized total proteins, irrespective of phosphorylation state. To determine protein phosphorylation, the intensity of phosphorylated signals was normalized to that of total protein. To determine protein abundance, total protein levels were normalized to $\alpha$-tubulin. For SREBP-1c processing, mature (cleaved) SREBP-1c was normalized to total SREBP-1c (precursor + mature).

\section{Subcellular Fractionation}

We isolated nuclear and cytoplasmic fraction from cellular extracts using the NE-PER Nuclear and Cytoplasmic Extraction Kit (ThermoFisher Scientific) according to the manufacturer's instructions (https:/ /assets.thermofisher.com/TFS-Assets/LSG/manuals/ MAN0011398_NEPER_Nuc_Cytoplasmic_Extract _Reag_UG.pdf). Protein concentration in each fraction was measured using the bicinchoninic acid (BCA) method. Nuclear and cytoplasmic fractions were subjected to immunoblotting and probed for SREBP-1c, GAPDH, or lamin-A/C, as described previously.

\section{RNA Extraction and Quantitative Real-Time PCR}

Total RNA was extracted from BMEC using the TRI-Reagent (Sigma-Aldrich), according to the manufacturer's instructions (https://www.sigmaaldrich.com/ technical-documents/protocols/biology/tri-reagent .html). RNA yield and quality were quantified by measuring absorbance at 260 and 260/280 nm, respectively, using a Take-3 multi-volume plate in a microplate spectrophotometer (BioTek, Winooski, VT). RNA integrity was determined by bleach agarose gel electrophoresis as described in Aranda et al. (2012). Total RNA (1 $\mu \mathrm{g})$ was reverse-transcribed using the iScript cDNA synthesis kit (Bio-Rad Laboratories) in a T100 thermal cycler (Bio-Rad Laboratories), according to the manufacturer's instructions (https://www.bio-rad.com/webroot/ web/pdf/lsr/literature/4106228.pdf).

Quantitative real-time PCR (qPCR) was performed using SsoAdvanced Universal SYBR green (Bio-Rad Laboratories) and gene-specific primers in a CFX96 Touch Real Time PCR System (Bio-Rad Laboratories). Primers were designed using Primer-Blast (Ye et al.,
2012). For validation of each primer, we performed melting curve analyses, determined amplicon length by agarose gel electrophoresis, determined optimal annealing temperature, and tested reaction efficiency using a dilution curve according to Taylor et al. (2010). The list of primers used are provided in Table 1. Samples for each experimental condition were run in triplicate. The reaction efficiency for each gene was $100 \pm 10 \%$ using a 10-fold serial dilution (Supplemental Figure S1; http: //dx.doi.org/10.3168/jds.2019-16343). We determined relative gene expression using the $\Delta \Delta \mathrm{Cq}$ method from the geometric mean of 2 reference genes $(A T C B$ and $R P L P O)$, selected from a group of 5 candidate reference genes under representative experimental conditions using geNorm (Hellemans et al., 2007). The results of the geNorm analysis are in Supplemental Figure S2 (http:/ /dx.doi.org/10.3168/jds.2019-16343). Using the $\Delta \Delta$ Cq method, it is possible that small differences in efficiency between reference and target genes could lead to slight under- or overestimation of treatment differences. Experiments were performed using CFX Maestro Software (Bio-Rad Laboratories).

\section{Statistical Analysis}

Data are presented as least squares means \pm standard error of the mean. Results are from 6 replicates per treatment group from BMEC derived from 3 independent cows (2 dishes per cow) in a randomized complete block design. We analyzed data by ANOVA using PROC MIXED in SAS (version 9.4; SAS Institute Inc., Cary, NC). For glucose and acetate deprivation experiments, treatment means were separated by least square differences. For time course and dose-response experiments, treatments were compared with $0 \mathrm{~h}$ and $4 \mathrm{~m} M$ glucose, respectively, by least square differences. Treatment differences were considered significant at $P$ $<0.05$, and a statistical trend at $0.05 \leq P<0.10$.

\section{RESULTS}

\section{Effect of A76 on AMPK Activation}

To investigate the role of AMPK in lipid and lactose synthesis by BMEC, we treated cells with $100 \mu M$ of A76, a new allosteric activator of AMPK. Unlike other pharmacological agents, A76 directly activates AMPK by mimicking both effects of AMP (i.e., allosteric activation and inhibition of dephosphorylation; Göransson et al., 2007). We confirmed lactogenic differentiation of BMEC by assessing the expression of mammary-specific milk protein genes $\alpha_{S 1}$ casein and $\alpha$-lactalbumin after $4 \mathrm{~d}$ of treatment with lactogenic hormones (data not shown). We assessed AMPK activation by measuring 


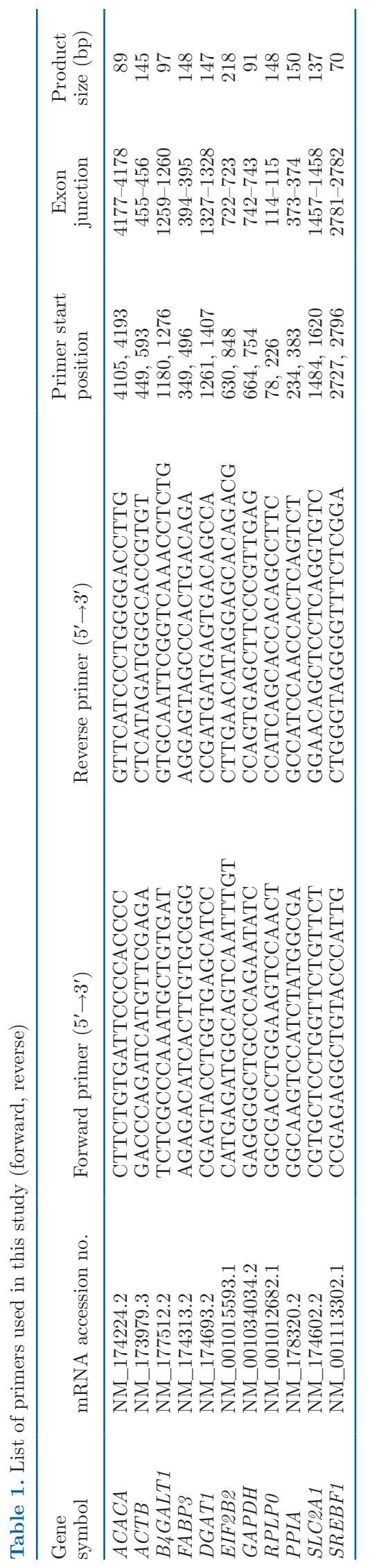

phosphorylation of AMPK $\alpha$ Thr172 and 2 downstream targets: ACC Ser79 and TSC2 Ser1387 (Gwinn et al., 2008). We found that AMPK $\alpha$ Thr172 phosphorylation was unaffected by incubation of BMEC in a medium containing $100 \mu M$ A76 (Figure 1A). In contrast, the phosphorylation of ACC at Ser79 and TSC2 at Ser1387 was $144 \%(P=0.015)$ and $26 \%(P=0.048)$ higher, respectively, in cells treated with A76 for $16 \mathrm{~h}$ compared with vehicle (Figures $1 \mathrm{~B}$ and $\mathrm{C}$ ). This finding was not unexpected, because ACC Ser79 phosphorylation is a more sensitive marker of AMPK activity than AMPKo Thr172 phosphorylation by this compound in intact cells (Göransson et al., 2007). Thus, treatment of BMEC with A76 activated AMPK in BMEC.

\section{Effect of AMPK Activation on De Novo Lipid Synthesis and Lipogenic Gene Expression}

To determine the effect of AMPK activation on de novo lipid synthesis, we measured the incorporation of radioactive acetate into total cellular lipids in BMEC treated with A76 or vehicle for $16 \mathrm{~h}$. Activation of AMPK by A76 decreased lipid synthesis in BMEC ( $P$ $=0.04$; Figure $2 \mathrm{~A}$ ).

It is known that AMPK exerts metabolic control on lipid synthesis by modulating the expression of lipogenic genes. To test this possibility, we measured the expression of key lipogenic genes in response to treatment with $\mathrm{A} 76$ for $16 \mathrm{~h}$ using qPCR. The mRNA expression of ACC, encoded by $A C A C A$, was not affected by A76 (Figure 2B). In addition to de novo fatty acid synthesis, BMEC take up preformed fatty acids for incorporation into milk lipids. FABP3 is one of the most abundant isoforms of a family of intracellular lipid-binding proteins that bind reversibly to fatty acids to facilitate their intracellular trafficking and metabolism in the lactating bovine mammary gland (Specht et al., 1996; Bionaz and Loor, 2008). FABP3 mRNA expression was $60 \%$ higher in A76-treated cells compared with control cells $(P=0.04$; Figure $2 \mathrm{C})$, consistent with a previous study in MAC-T cells using AICAR (McFadden and Corl, 2009). Activation of AMPK by A76 did not significantly affect $D G A T 1$ or $S R E B F 1$ mRNA expression (Figure 2D and E).

\section{Effect of AMPK Activation on SREBP-1c Protein Abundance, Proteolytic Processing, and Subcellular Localization}

To examine the potential role of SREBP-1c in mediating the effect of AMPK on lipid synthesis, we investigated the effect of A76 on SREBP-1c abundance, proteolytic processing, and subcellular localization in BMEC. As shown in Figure 3A, treatment with 

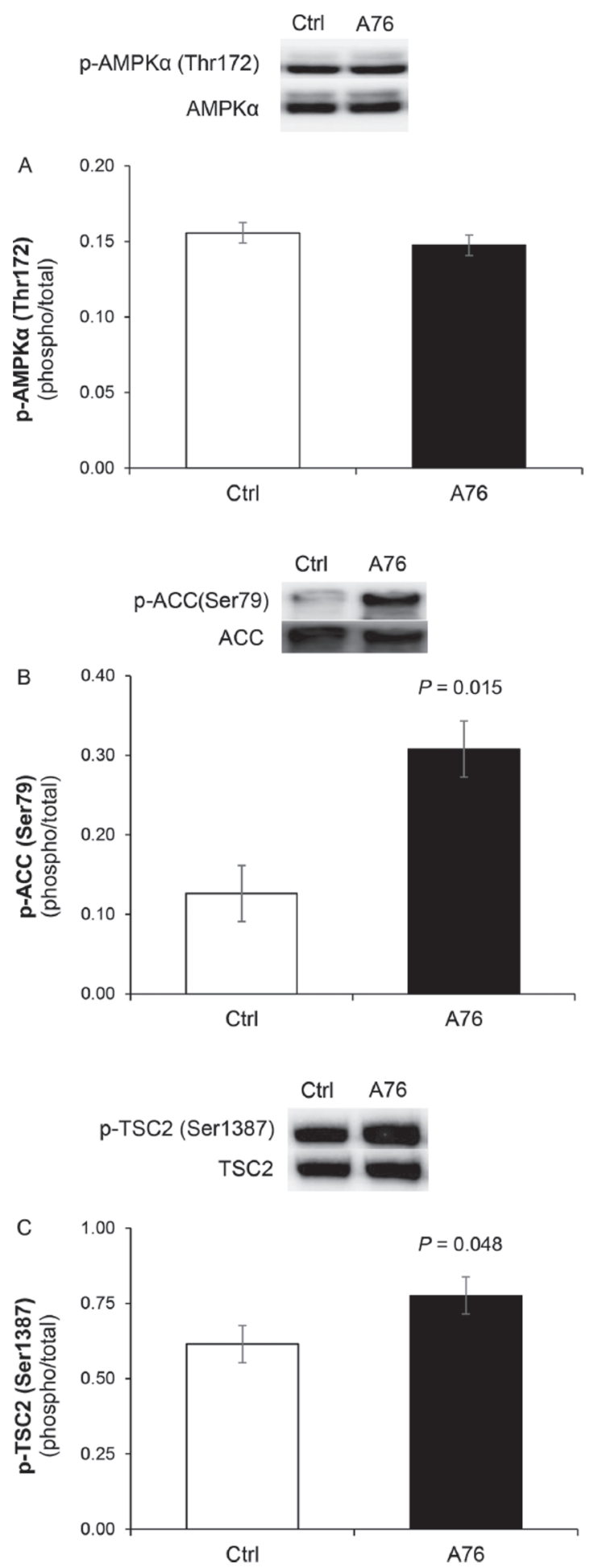

Figure 1. Effect of allosteric activator A76 on AMPK activation. Lactogenic differentiated bovine mammary epithelial cells were treated with $100 \mu M$ A-769662 (A76) or vehicle control (Ctrl) for $16 \mathrm{~h}$. Phosphorylation (p) of (A) AMPKo at Thr172, (B) ACC at Ser79, and (C) TSC2 at Ser1387 was measured by immunoblot analysis. The densities of phosphorylated AMPK, ACC, and TSC2 were normalized to total AMPK, ACC, and TSC2 protein levels, respectively. Representative immunoblots are shown. Data are presented as LSM \pm SEM.
A76 tended to increase total (precursor plus mature) SREBP-1c protein abundance by $30 \%(P=0.077)$. The proportion of mature SREBP-1c to total SREBP$1 \mathrm{c}$, including precursor and mature forms, from A76treated cells exhibited $19 \%$ less expression compared with vehicle $(P=0.0005$; Figure $3 \mathrm{~B})$. We also found a nominal decline in the abundance of mature SREBP$1 \mathrm{c}$ in both nuclear and cytoplasmic fractions in cells treated with A76 compared with vehicle control, but the differences were not significant $(P=0.12$ and $P=$ 0.56, respectively; Figure 3C).

\section{Effect of AMPK Activation on Lactose Synthesis and Gene Expression}

To investigate the potential role of AMPK in regulation of lactose synthesis, we measured radioactive glucose incorporation into lactose. Treatment of BMEC with A76 significantly decreased the incorporation of radiolabelled glucose into lactose $(P=0.01$; Figure 4A). Therefore, we measured the expression of key genes involved in lactose synthesis. The transcript abundance of GLUT1, encoded by SLC2A1, was $21 \%$ lower in BMEC treated with A76 compared with vehicle (Figure 4B). Expression of $\beta$-1,4-galactosyltransferase (B4GALT1), which forms lactose synthase in a complex with $\alpha$-lactalbumin, was not affected by AMPK activation (Figure 4C).

\section{Effect of Glucose and Acetate Deprivation on AMPK Activation}

Both acetate and glucose are sources of energy used by BMEC for maintenance functions and the synthesis of milk components. To examine the effect of energyyielding substrate depletion on AMPK activation, we measured the phosphorylation of AMPK and its substrates in BMEC deprived of glucose or acetate, alone or in combination, for $4 \mathrm{~h}$. Deprivation of glucose or acetate promoted phosphorylation of AMPK $\alpha$ at Thr172 by $84 \%(P=0.01)$ or $58 \%(P=0.05)$, respectively, compared with vehicle (Figure 5A). Although deprivation of energy substrates, to a certain extent, nominally increased phosphorylation of TSC2 at Ser1387, only deprivation of both glucose and acetate caused a significant change to TSC2 $(P=0.03$; Figure $5 \mathrm{~B})$. Thus, a shortage of acetate and glucose in BMEC caused the activation of AMPK.

\section{Time Course of AMPK Activation in Response to Glucose Deprivation}

We examined the time-dependent activation of AMPK by glucose deprivation. We measured the phosphoryla- 
tion of AMPK and its downstream targets in BMEC deprived of glucose for $0,1,2$, or $4 \mathrm{~h}$. Phosphorylation of AMPK increased gradually with time, up to $62 \%$ ( $P$ $=0.01$ ) by $4 \mathrm{~h}$ of glucose deprivation compared with 0 h (Figure 6A). Phosphorylation of ACC at Ser79 and TSC2 at Ser1387 followed a similar temporal pattern to that of AMPK at Thr172 upon glucose withdrawal. At least $2 \mathrm{~h}$ of glucose deprivation were required AMPKmediated phosphorylation of ACC $(P=0.02)$, whereas
TSC2 phosphorylation was significantly different only after $4 \mathrm{~h}$ of glucose deprivation $(P=0.001$; Figure $6 \mathrm{~B}$ and $\mathrm{C}$ ).

\section{Dose Response of Glucose Deprivation on AMPK Activation}

To assess the sensitivity of AMPK to glucose levels, we measured the phosphorylation of AMPK and its
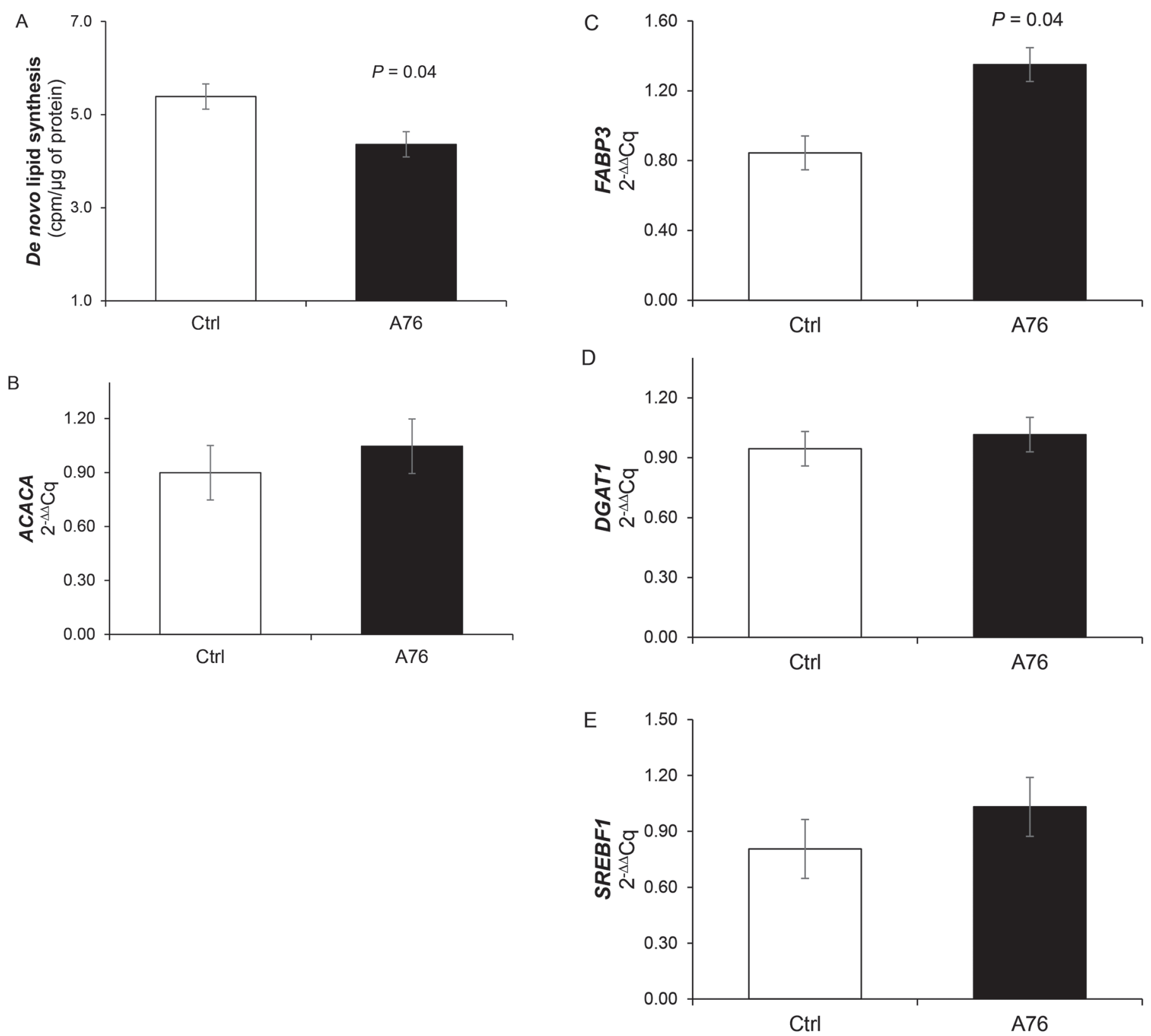

Figure 2. Effect of AMPK activation on de novo lipid synthesis and lipogenic gene expression. Cells were treated with $100 \mu M$ A-769662 (A76) or vehicle control (Ctrl) for $16 \mathrm{~h}$. (A) Incorporation of ${ }^{3} \mathrm{H}$-acetate into total lipids was measured by liquid scintillation counting. The mRNA expression of genes encoding (B) acetyl-CoA carboxylase a $(A C A C A)$, (C) fatty acid binding protein 3 (FABP3), (D) diacylglycerol O-acyltransferase 1 (DGAT1), and (E) sterol regulatory element binding protein-1 (SREBF1) was evaluated using quantitative PCR. Data are presented as LSM \pm SEM. 
Treatment: Ctrl A76

SREBP-1c Precursor

SREBP-1c Mature

a-Tubulin
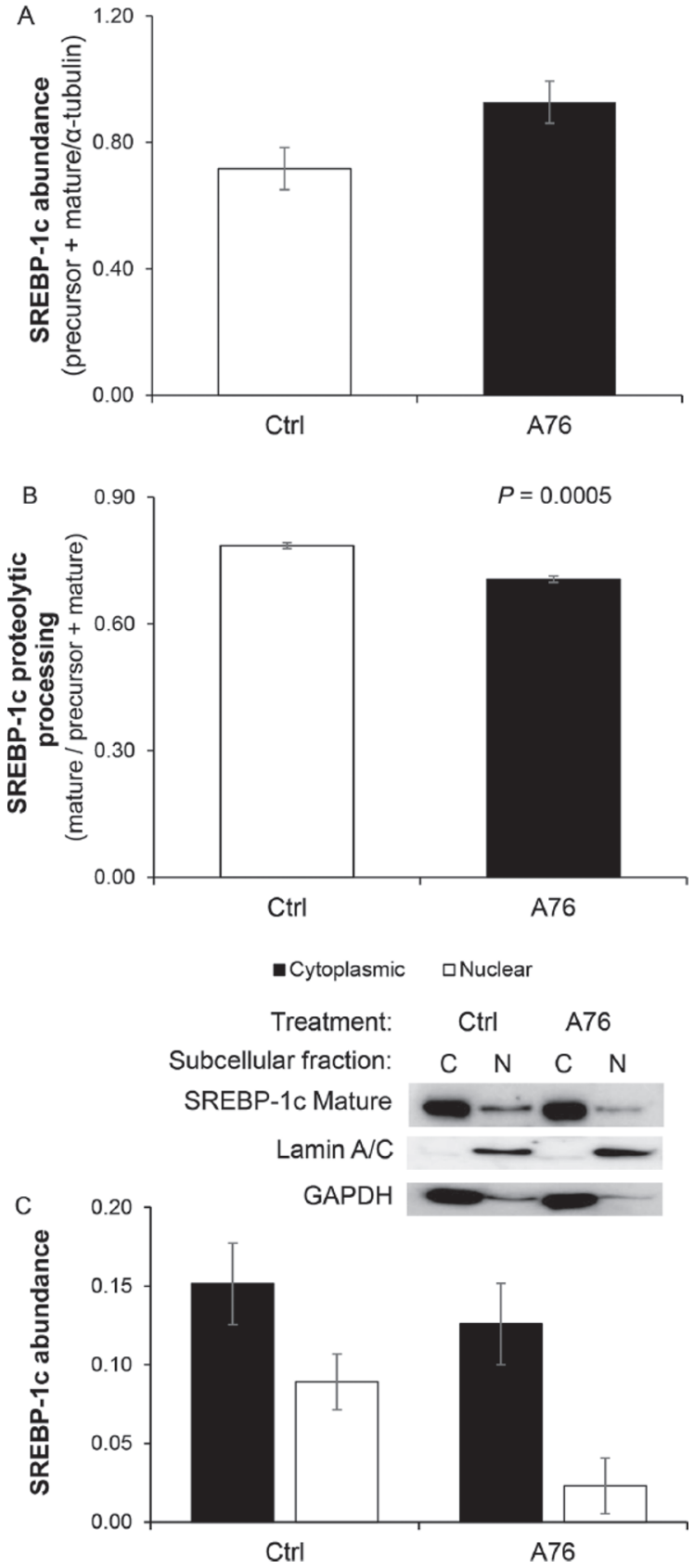

Figure 3. Effect of AMP-activated protein kinase activation on SREBP-1c protein abundance, proteolytic processing, and subcellular localization. Lactogenic differentiated bovine mammary epithelial cells were treated with $100 \mu \mathrm{M}$ A-769662 (A76) or vehicle control (Ctrl) for $16 \mathrm{~h}$. The abundance of (A) total SREBP-1c, (B) mature SREBP-1c proportion, and $(\mathrm{C})$ nuclear and cytoplasmic SREBP-1c were measured by immunoblot analysis. The densities of nuclear and cytoplasmic SREBP-1c was normalized to lamin-A/C and GAPDH protein levels, respectively. Representative immunoblots are shown. Data are presented as LSM $\pm \mathrm{SEM}$. C = cytoplasmic; $\mathrm{N}=$ nuclear. downstream targets in BMEC treated without or with 1,2 , or $4 \mathrm{~m} M$ glucose for $4 \mathrm{~h}$. Cells exposed to low glucose concentrations did not have a significant change in AMPK phosphorylation compared with $4 \mathrm{~m} M$ glucose; we observed a significant difference only upon complete glucose deprivation $(P=0.05$; Figure $7 \mathrm{~A})$. When re-
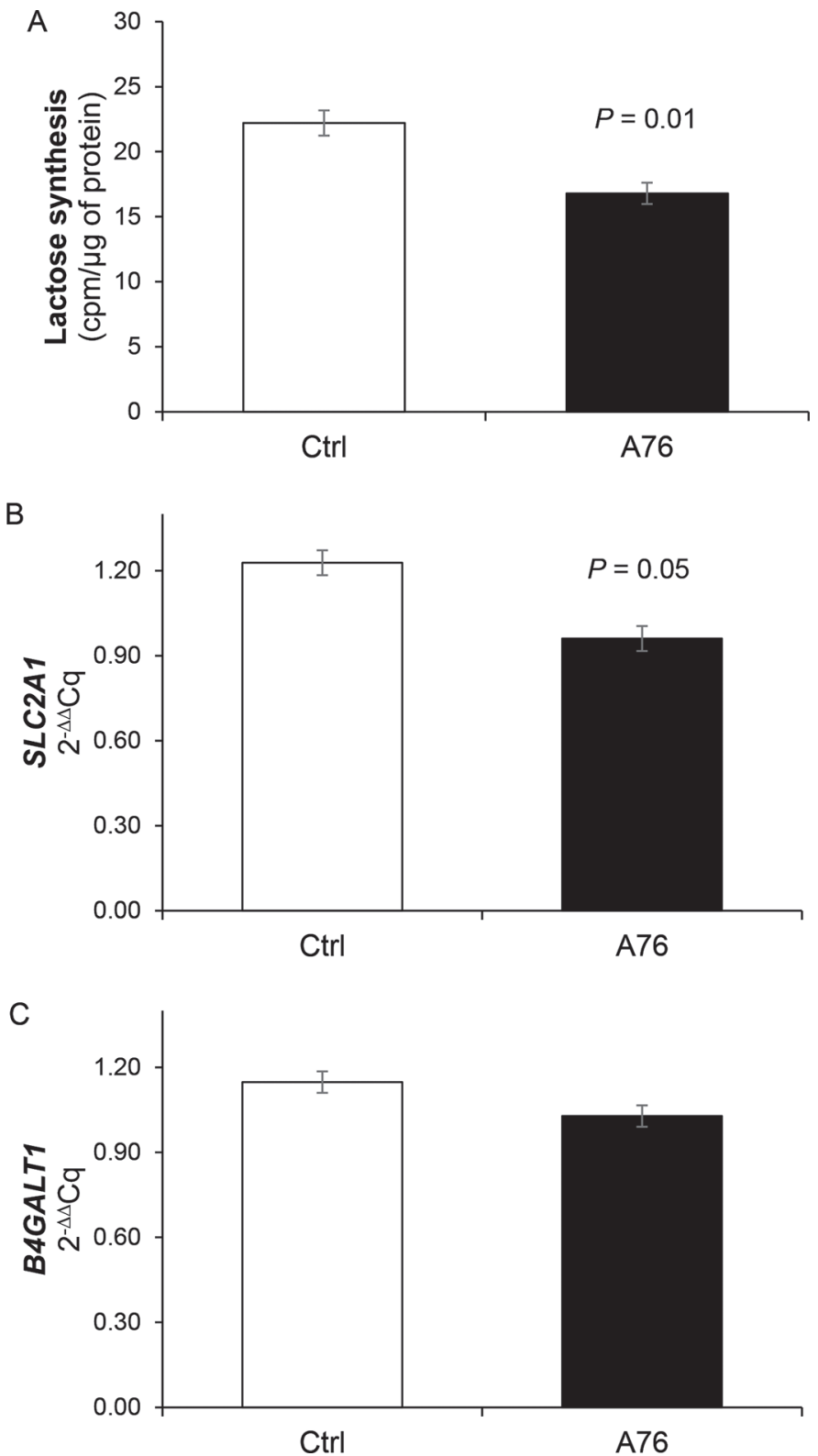

Figure 4. Effect of AMP-activated protein kinase activation on lactose synthesis and gene expression. Lactogenic differentiated bovine mammary epithelial cells were treated with $100 \mu M$ A-769662 (A76) or vehicle control (Ctrl) for $16 \mathrm{~h}$. (A) Incorporation of ${ }^{14} \mathrm{C}$-glucose into total lactose was measured by liquid scintillation counting. $\mathrm{cpm}=$ counts per minute. The mRNA expression of genes encoding (B) solute carrier family 2 member 1 (SLC2A1) and (C) $\beta$-1,4-galactosyltransferase (B4GALT1) was evaluated by using quantitative PCR. Data are presented as $\mathrm{LSM} \pm \mathrm{SEM}$. 
ducing glucose levels from 4 to 2 or $1 \mathrm{mM}$, we found no change in the phosphorylation of AMPK targets ACC and TSC2. However, glucose deprivation $(0 \mathrm{mM})$ increased the phosphorylation of ACC and TSC2 by $249 \%(P=0.01)$ and $50 \%(P=0.003)$, respectively

A

Treatment: $\quad$ Ctrl $-\mathrm{Glc}-\mathrm{Ac}-\mathrm{Glc} / \mathrm{Ac}$

p-AMPKa (Thr172)

AMPKa
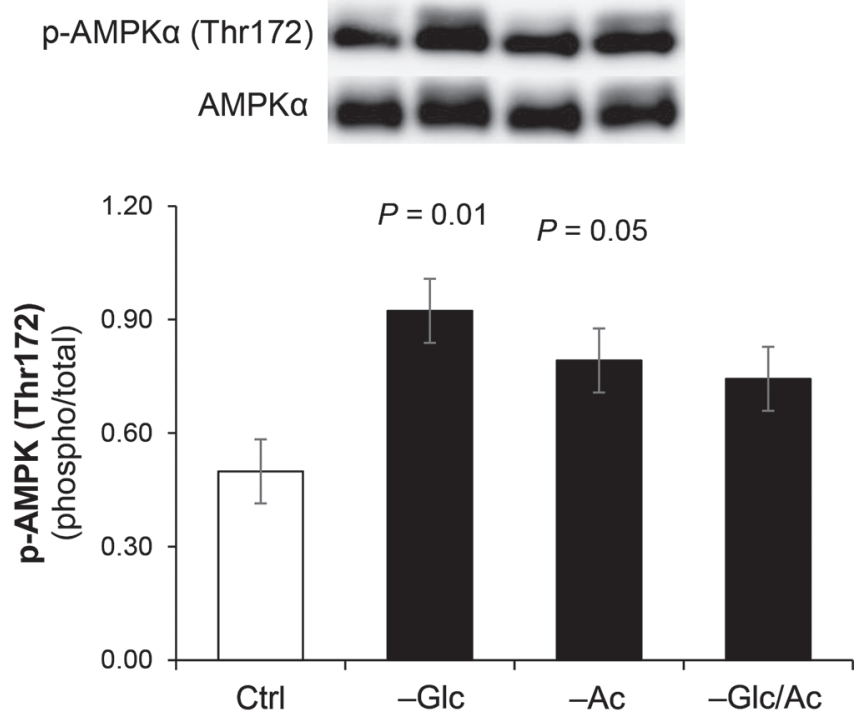

B

Treatment: Ctrl -Glc -Ac-Glc/Ac

p-TSC2 (Ser1387)

TSC2

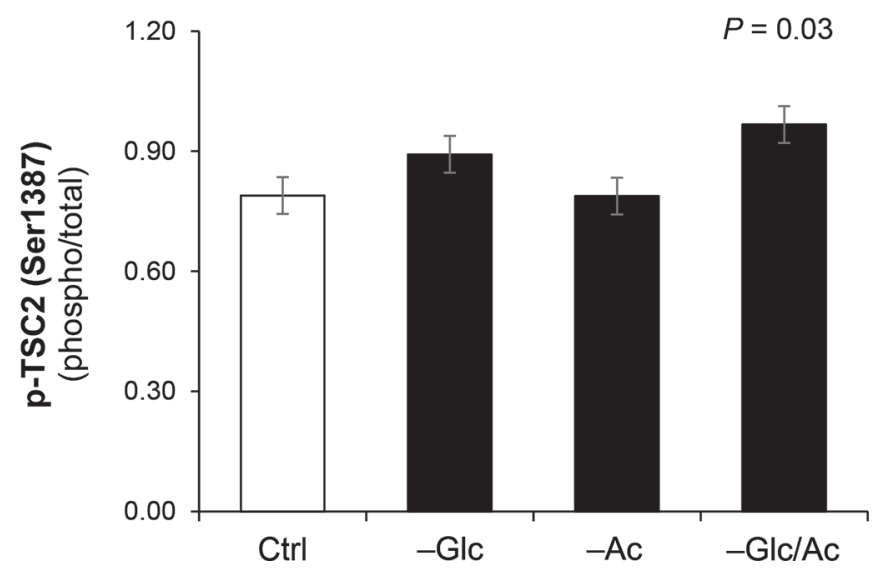

Figure 5. Effect of glucose and acetate deprivation on AMPactivated protein kinase (AMPK) activation. Lactogenic differentiated bovine mammary epithelial cells were incubated in Dulbecco's modified Eagle medium (DMEM) containing $4 \mathrm{~m} M$ glucose and $1 \mathrm{~m} M$ sodium acetate (Ctrl) or DMEM without glucose $(-\mathrm{Glc})$, acetate $(-\mathrm{Ac})$, or both glucose and acetate (-Glc/Ac) for $4 \mathrm{~h}$. Phosphorylation (p) of (A) AMPKa at Thr172 and (B) TSC2 at Ser1387 was measured by immunoblot analysis. The densities of phosphorylated AMPK and TSC2 were normalized to total AMPK and TSC2 protein levels, respectively. Representative immunoblots are shown. Data are presented as $\mathrm{LSM} \pm \mathrm{SEM}$.
A

Time (h): $\quad \begin{array}{llll}0 & 1 & 2 & 4\end{array}$

p-AMPK (Thr172)

AMPK

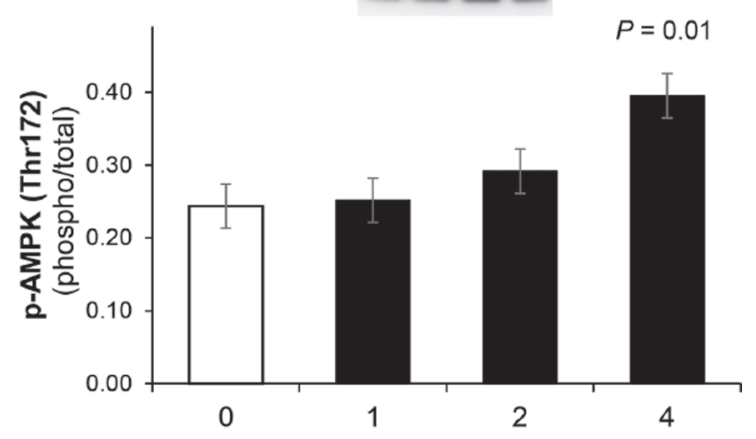

B

Time (h): $\quad \begin{array}{llll}0 & 1 & 2 & 4\end{array}$

p-ACC (Ser79)

ACC $\mathrm{aratan}$

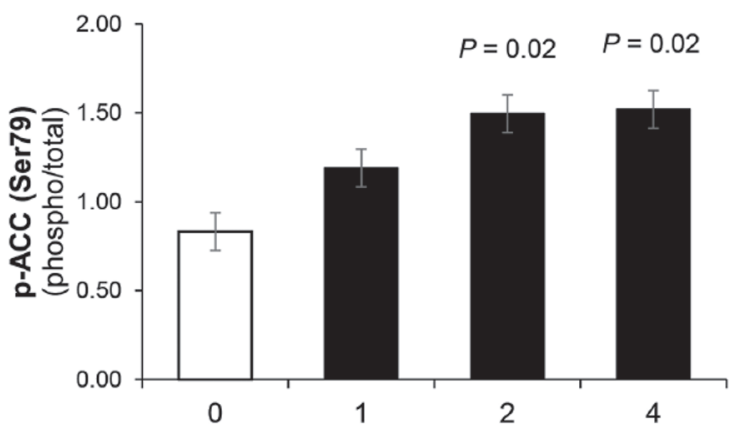

C
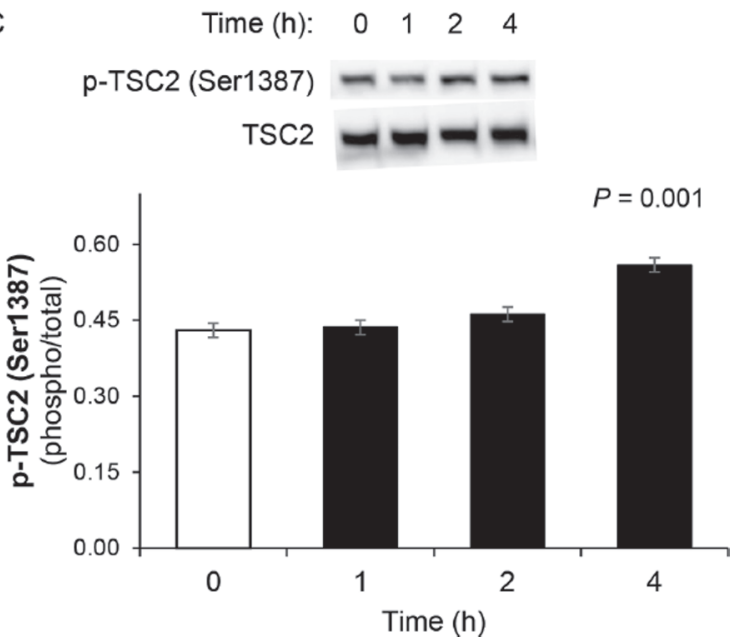

Figure 6. Time course of AMP-activated protein kinase (AMPK) activation in response to glucose deprivation. Lactogenic differentiated bovine mammary epithelial cells were incubated in Dulbecco's modified Eagle medium without glucose for 0, 1, 2, or $4 \mathrm{~h}$. Phosphorylation (p) of (A) AMPKo at Thr172, (B) ACC at Ser79, and (C) TSC2 at Ser1387 was measured by immunoblot analysis. The densities of phosphorylated AMPK, TSC2, and ACC were normalized to total AMPK, TSC2, and ACC protein levels, respectively. Representative immunoblots are shown. Data are presented as LSM \pm SEM. 
(Figure 7B and C). Thus, complete glucose deprivation was required to trigger AMPK activation in BMEC, suggesting that other metabolic fuels maintain ATPAMP levels at low glucose levels.

\section{DISCUSSION}

Previous studies have established a potential role for AMPK in the control of mammary nutrient metabolism and milk component synthesis in BMEC. Burgos et al. (2013) demonstrated that AMPK activation inhibits mammary protein synthesis through mTOR complex 1 (mTORC1) in MAC-T cells. McFadden and Corl (2009) showed that AMPK activation blunted de novo lipid synthesis in MAC-T cells treated with the AMP analog AICAR, but the mechanisms remained elusive. In this study, we used a novel allosteric activator of AMPK to confirm this observation, and extended it by demonstrating that SREBP-1c may mediate the effect of AMPK on lipid synthesis by modulating SREBP-1c proteolytic processing and nuclear translocation. Collectively, these studies support an important role for AMPK in the regulation of lipid and lactose synthesis in BMEC.

The mechanism by which AMPK activation reduces de novo lipid synthesis in BMEC has not been fully elucidated. In MAC-T cells, the inhibition of de novo lipid synthesis by AICAR was accompanied by increased mRNA expression of several lipogenic genes, including SREBF1, FASN, and FABP3 by 66,38 , and $56 \%$, respectively (McFadden and Corl, 2009). In partial agreement, we found that the mRNA expression of $F A B P 3$ increased by $60 \%$ in response to A76, whereas the expression of other lipogenic genes (DGAT1 and $A C A C A$ ) did not differ significantly from control. In the present study, treatment of BMEC with A76 increased the phosphorylation of ACC $\alpha$ by $144 \%$, but the mRNA expression of $A C A C A$ was unaffected, consistent with a previous study in MAC-T cells by McFadden and Corl (2009). The same authors also observed a significant decrease in ACC activity in response to increased $\mathrm{ACC} \alpha$ phosphorylation (McFadden and Corl, 2009). The reduction in ACC activity most likely contributed to the significant decrease in total lipid synthesis in response to treatment of A76 in our study. However, definitive proof of this notion would require a fatty acid profile of total cellular lipids. McFadden and Corl (2009) previously reported differences in AMPK subunit expression between mammary gland tissue and MAC-T cell, which we speculate could explain in part the few discrepancies between studies.

Activation of AMPK modulates SREBP-1c expression by inhibiting the trafficking of SREBP to the nucleus, thereby controlling its lipogenic activity. We
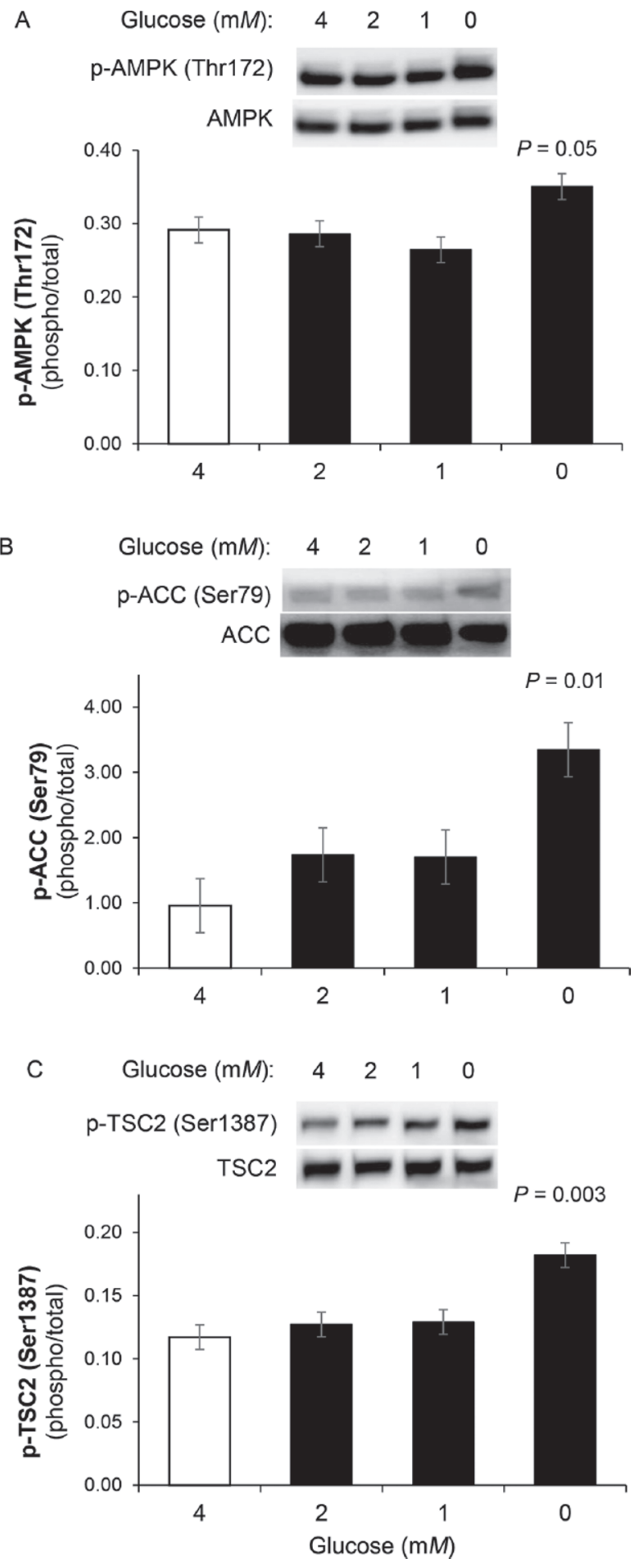

Figure 7. Dose response of glucose deprivation on AMP-activated protein kinase (AMPK) activation. Dose response of glucose on phosphorylation (p) of AMPK, TSC2, and ACC in bovine mammary epithelial cells. Cells were incubated in Dulbecco's modified Eagle medium containing $0,1,2$, or $4 \mathrm{~m} M$ glucose for $4 \mathrm{~h}$. Phosphorylation of (A) AMPKo at Thr172, (B) ACC at Ser79, and (C) TSC2 at Ser1387 was measured by immunoblot analysis. The densities of phosphorylated AMPK, TSC2, and ACC were normalized to total AMPK, TSC2, and ACC protein levels, respectively. Representative immunoblots are shown. Data are presented as LSM \pm SEM. 
showed that activation of AMPK by A76 tended to increase SREBP-1c protein abundance, but the proportion of mature SREBP-1c actually decreased. Quan et al. (2013) found that activation of AMPK suppressed SREBP-1c nuclear translocation and repressed SREBP$1 \mathrm{c}$ target gene expression, resulting in decreased lipogenesis and lipid accumulation in HepG2 cells and primary hepatocytes. When activated by metformin, AMPK suppressed SREBP-1c cleavage and nuclear translocation in hepatocytes (Li et al., 2011). However, we found that the established SREBP-1c target genes measured in our study did not change or increased, similar to previous findings in AICAR-treated MAC-T cells by McFadden and Corl (2009). Thus, the SREBP-1c targets that mediate AMPK inhibition of lipid synthesis have yet to be identified. In addition, whether other lipogenic transcription factors such as LXR $\alpha$ cooperate with SREBP-1c to regulate lipogenesis in BMEC downstream of AMPK remains to be determined.

Activation of AMPK can regulate genes that control glucose metabolism (Magnoni et al., 2012). Treatment of BMEC with A76 reduced the mRNA expression of the gene encoding for GLUT1. In contrast, Zhang et al. (2011) reported that intracellular glucose levels (which were interpreted to indicate heightened glucose uptake) and the mRNA abundance of GLUT1 and glycogen synthase increased after incubation of goat mammary epithelial cells with AICAR. The change in intracellular glucose levels detected in response to AICAR could also be explained by a decrease in glucose use. In this regard, we found that lactose synthesis was markedly reduced by the activation of AMPK. Further research is needed to identify the mechanisms by which AMPK inhibits lactose synthesis in BMEC.

We know that AMPK is sensitive to cellular energy status in various cell types, and the decrease in energy charge activates AMPK. In dairy cows, mammary cells use acetate and glucose as sources of energy. In earlier work, Appuhamy et al. (2014) tested the effect of essential amino acids in the absence or presence of acetate $(5$ $\mathrm{m} M)$ or glucose $(17.5 \mathrm{mM})$ on mTORC1 signaling and protein synthesis in MAC-T cells and bovine mammary tissue slices. They found that the provision of essential amino acids with or without glucose reduced AMPK $\alpha$ Thr172 phosphorylation relative to nutrient-deprived MAC-T cells. In the present study, glucose and acetate differentially affected the phosphorylation of AMPK and its downstream targets; glucose deprivation had the greatest effect on AMPK $\alpha$ Thr172 phosphorylation. Dose-response experiments revealed that AMPK was activated when glucose was absent from the culture medium. Furthermore, time course experiments revealed that AMPK responded only to long-term depletion of glucose supply. These results suggest that oxidation of other metabolic fuels, possibly amino acids, can allow BMEC to cope with low glucose levels or short-term decrease in glucose supply. There is evidence to suggest that AMPK senses intracellular glucose levels directly, in addition to cellular energy stress (Lin and Hardie, 2018).

This study had certain limitations. The use of pharmacological agents allowed for the assessment of AMPK function on milk components synthesis in vitro. In this context, it is important to note that only complete deprivation of glucose induced activation of AMPK in cultured BMEC. Therefore, the physiological relevance of AMPK as a sensor of cellular energy status and its role in the control of milk components synthesis will require further studies in vivo. This could be tested by assessing AMPK activation in mammary biopsies from cows under conditions of negative energy balance or during feed restriction. Our data suggest that AMPK may be involved in controlling lactose synthesis as measured by radioactive glucose present in lactose$\mathrm{Mg}(\mathrm{OH})_{2}$ precipitate, but this method lacks specificity for lactose. Further evidence for a role of AMPK in lactose synthesis will require confirmation of changes in lactose content using analyte-specific assays.

\section{CONCLUSIONS}

Allosteric activation of AMPK by A76 decreased the synthesis of both lipid and lactose in BMEC. The AMPK-induced inhibition of lipid synthesis in BMEC was accompanied by increased phosphorylation of ACC $\alpha$ and expression of FABP3. However, AMPK activation reduced the proteolytic processing of SREBP1, which would be expected to reduce lipogenic gene expression. Activation of AMPK also reduced lactose synthesis and SLC2A1 mRNA expression. The mechanisms that mediate the effect of AMPK on lactose and lipid synthesis remain to be elucidated. Deprivation of glucose and acetate led to activation of AMPK, as judged by phosphorylation at Thr172 of its $\alpha$ subunit and its downstream targets, but changes occurred only with a medium completely devoid of glucose and after 2 to $4 \mathrm{~h}$, suggesting that other metabolic fuels could temporarily make up for inadequate glucose supply. The physiological relevance of AMPK as a sensor of cellular energy status and its role in the control of milk component synthesis will require metabolic studies in dairy cows.

\section{ACKNOWLEDGMENTS}

J. Huang was supported by a Marian and Ralph Sketch Fellowship. M.-A. Guesthier was supported in part by a scholarship Op ${ }^{+}$LAIT Regroupement Bourses 
Complementaries. This work was supported by a new researcher's startup grant from Fonds de recherche du Québec-Nature et technologies to S. Burgos (NC205408). The lamin-A/C hybridoma supernatant (developed by G. E. Morris) was obtained from the Developmental Studies Hybridoma Bank, created by the National Institute of Child Health and Human Development (NICHD) and maintained at the University of Iowa.

\section{REFERENCES}

Appuhamy, J. A., W. Nayananjalie, E. England, D. Gerrard, R. Akers, and M. Hanigan. 2014. Effects of AMP-activated protein kinase (AMPK) signaling and essential amino acids on mammalian target of rapamycin (mTOR) signaling and protein synthesis rates in mammary cells. J. Dairy Sci. 97:419-429.

Aranda, P. S., D. M. LaJoie, and C. L. Jorcyk. 2012. Bleach gel: A simple agarose gel for analyzing RNA quality. Electrophoresis 33:366-369.

Bionaz, M., and J. J. Loor. 2008. Gene networks driving bovine milk fat synthesis during the lactation cycle. BMC Genomics 9:366.

Burgos, S. A., M. Dai, and J. P. Cant. 2010. Nutrient availability and lactogenic hormones regulate mammary protein synthesis through the mammalian target of rapamycin signaling pathway. J. Dairy Sci. 93:153-161.

Burgos, S. A., J. J. Kim, M. Dai, and J. P. Cant. 2013. Energy depletion of bovine mammary epithelial cells activates AMPK and suppresses protein synthesis through inhibition of mTORC1 signaling. Horm. Metab. Res. 45:183-189.

Carlson, C. A., and K. H. Kim. 1973. Regulation of hepatic acetyl coenzyme A carboxylase by phosphorylation and dephosphorylation. J. Biol. Chem. 248:378-380.

Garbe, J. C., S. Bhattacharya, B. Merchant, E. Bassett, K. Swisshelm, H. S. Feiler, A. J. Wyrobek, and M. R. Stampfer. 2009. Molecular distinctions between stasis and telomere attrition senescence barriers shown by long-term culture of normal human mammary epithelial cells. Cancer Res. 69:7557-7568.

Göransson, O., A. McBride, S. A. Hawley, F. A. Ross, N. Shpiro, M. Foretz, B. Viollet, D. G. Hardie, and K. Sakamoto. 2007. Mechanism of action of A-769662, a valuable tool for activation of AMPactivated protein kinase. J. Biol. Chem. 282:32549-32560.

Guinard-Flament, J., E. Delamaire, S. Lemosquet, M. Boutinaud, and Y. David. 2006. Changes in mammary uptake and metabolic fate of glucose with once-daily milking and feed restriction in dairy cows. Reprod. Nutr. Dev. 46:589-598.

Gwinn, D. M., D. B. Shackelford, D. F. Egan, M. M. Mihaylova, A. Mery, D. S. Vasquez, B. E. Turk, and R. J. Shaw. 2008. AMPK phosphorylation of raptor mediates a metabolic checkpoint. Mol. Cell 30:214-226.

Hardie, D. G., and D. A. Pan. 2002. Regulation of fatty acid synthesis and oxidation by the AMP-activated protein kinase. Biochem. Soc. Trans. 30:1064-1070.

Hardie, D. G. 2011a. AMP-activated protein kinase-An energy sensor that regulates all aspects of cell function. Genes. Dev. 25:18951908.
Hardie, D. G. 2011b. Energy sensing by the AMP-activated protein kinase and its effects on muscle metabolism. Proc. Nutr. Soc. 70:92-99.

Hardie, D. G., B. E. Schaffer, and A. Brunet. 2016. AMPK: An energy-sensing pathway with multiple inputs and outputs. Trends Cell Biol. 26:190-201.

Hawley, S. A., M. Davison, A. Woods, S. P. Davies, R. K. Beri, D. Carling, and D. G. Hardie. 1996. Characterization of the AMPactivated protein kinase kinase from rat liver and identification of threonine 172 as the major site at which it phosphorylates AMPactivated protein kinase. J. Biol. Chem. 271:27879-27887.

Hellemans, J., G. Mortier, A. De Paepe, F. Speleman, and J. Vandesompele. 2007. qBase relative quantification framework and software for management and automated analysis of real-time quantitative PCR data. Genome Biol. 8:R19.

Kwon, S.-Y., R. A. Bernhard, and T. A. Nickerson. 1981. Recovery of lactose from aqueous solutions: Precipitation with magnesium chloride and sodium hydroxide. J. Dairy Sci. 64:396-406.

Li, Y., S. Xu, M. M. Mihaylova, B. Zheng, X. Hou, B. Jiang, O. Park, Z. Luo, E. Lefai, and J. Y.-J. Shyy. 2011. AMPK phosphorylates and inhibits SREBP activity to attenuate hepatic steatosis and atherosclerosis in diet-induced insulin-resistant mice. Cell Metab. $13: 376-388$

Lin, S. C., and D. G. Hardie. 2018. AMPK: Sensing glucose as well as cellular energy status. Cell Metab. 27:299-313.

Magnoni, L. J., Y. Vraskou, A. P. Palstra, and J. V. Planas. 2012. AMP-activated protein kinase plays an important evolutionary conserved role in the regulation of glucose metabolism in fish skeletal muscle cells. PLoS One 7:e31219.

McFadden, J. W., and B. A. Corl. 2009. Activation of AMP-activated protein kinase (AMPK) inhibits fatty acid synthesis in bovine mammary epithelial cells. Biochem. Biophys. Res. Commun. 390:388-393.

Mellenberger, R. W., D. E. Bauman, and D. R. Nelson. 1973. Metabolic adaptations during lactogenesis. Fatty acid and lactose synthesis in cow mammary tissue. Biochem. J. 136:741-748.

Quan, H. Y., S. J. Kim, H. K. Jo, G. W. Kim, and S. H. Chung. 2013. Betulinic acid alleviates non-alcoholic fatty liver by inhibiting SREBP1 activity via the AMPK-mTOR-SREBP signaling pathway. Biochem. Pharmacol. 85:1330-1340.

Specht, B., N. Bartetzko, C. Hohoff, H. Kuhl, R. Franke, T. Borchers, and F. Spener. 1996. Mammary derived growth inhibitor is not a distinct protein but a mix of heart-type and adipocyte-type fatty acid-binding protein. J. Biol. Chem. 271:19943-19949.

Taylor, S., M. Wakem, G. Dijkman, M. Alsarraj, and M. Nguyen. 2010. A practical approach to RT-qPCR-publishing data that conform to the MIQE guidelines. Methods 50:S1-S5.

Ye, J., G. Coulouris, I. Zaretskaya, I. Cutcutache, S. Rozen, and T. L. Madden. 2012. Primer-BLAST: A tool to design target-specific primers for polymerase chain reaction. BMC Bioinformatics 13:134.

Zhang, N., Q. Li, X. Gao, and H. Yan. 2011. Potential role of adenosine monophosphate-activated protein kinase in regulation of energy metabolism in dairy goat mammary epithelial cells. J. Dairy Sci. 94:218-222.

\section{ORCIDS}

S. A. Burgos @ https://orcid.org/0000-0003-0569-4307 